\title{
Correction to: LyP-1 Conjugated Nanoparticles for Magnetic Resonance Imaging of Triple Negative Breast Cancer
}

\author{
Abedelnasser Abulrob, ${ }^{1,2,3}$ Slavisa Corluka, ${ }^{1}$ Barbara Blasiak, ${ }^{4,5}$ B. Gino Fallone, ${ }^{6}$ \\ Dragana Ponjevic, ${ }^{7}$ John Matyas, ${ }^{7}$ Boguslaw Tomanek ${ }^{4,5,6}$ \\ ${ }^{1}$ Department of Cellular and Molecular Medicine, Faculty of Medicine, University of Ottawa, Ottawa, ON, Canada \\ ${ }^{2}$ Brain and Mind Research Institute, University of Ottawa, Ottawa, ON, Canada \\ ${ }^{3}$ Human Health Therapeutics Portfolio, National Research Council Canada, Ottawa, ON, K1A 0R6, Canada \\ ${ }^{4}$ Institute of Nuclear Physics, Polish Academy of Sciences, Krakow, Poland \\ ${ }^{5}$ Department of Clinical Neurosciences, University of Calgary, Calgary, Canada \\ ${ }^{6}$ Department of Oncology, University of Alberta, Edmonton, Canada \\ ${ }^{7}$ Department of Comparative Biology \& Experimental Medicine, Faculty of Veterinary Medicine, University of Calgary, Calgary, Canada
}

This article was updated to correct the spelling of B. Gino Fallone's name; it is correct as displayed above.

The online version of the original article can be found at https://doi.org/ $10.1007 / \mathrm{s} 11307-017-1140-4$

Correspondence to: Abedelnasser Abulrob; e-mail: Abedelnasser.abulrob@nrc.gc.ca, Boguslaw Tomanek; e-mail: tomanek@ualberta.ca 